\title{
A Cooperative Location Management Scheme for Mobile Ad Hoc Networks
}

\author{
Demin LI ${ }^{1}$, Jiacun WANG ${ }^{2}$, Liping ZHANG ${ }^{3}$, Hao LI ${ }^{1}$, Jie ZHOU ${ }^{4}$ \\ ${ }^{1}$ College of Information Science and Technology, Donghua University, Songjiang District, Shanghai, China \\ ${ }^{2}$ Department of Software Engineering, Monmouth University, West Long Branch, NJ, USA \\ ${ }^{3}$ College of Electronic and Electrical Engineering, Shanghai University of Engineering Science, \\ Songjiang District, Shanghai, China \\ ${ }^{4}$ School of Science, Donghua University, Songjiang District, Shanghai, China \\ Email:deminli@dhu.edu.cn, jwang@monmouth.edu,zhangliping@sues.edu.cn,haoli@mail.dhu.edu.cn \\ Received July 8, 2009; revised August 17, 2009; accepted September 22, 2009
}

\begin{abstract}
A mobile ad hoc network (MANET) is a kind of wireless ad hoc network. It is a self-configuring network of mobile routers connected by wireless links. Since MANETs do not have a fixed infrastructure, it is a challenge to design a location management scheme that is both scalable and cost-efficient. In this paper, we propose a cooperative location management scheme, called CooLMS, for MANETs. CooLMS combines the strength of grid based location management and pointer forwarding strategy to achieve high scalability and low signaling cost. An in-depth formal analysis of the location management cost of CooLMS is presented. In particular, the total location management cost of mobile nodes moving at variable velocity is estimated using the Gauss_Markov mobility model for the correlation of mobility velocities. Simulation results show CooLMS performs better than other schemes under certain circumstances.
\end{abstract}

Keywords: Ad hoc Network, Grid, Home Region, Location Management, Forwarding Pointer, Cost Estimation

\section{Introduction}

Mobile ad hoc networks consist of wireless hosts that communicate with each other in the absence of a fixed infrastructure. Some examples of the possible uses of ad hoc networks include soldiers on the battlefield, emergency disaster relief personnel, and networks of laptops. Routing a packet from a source to a destination in a mobile ad hoc network is challenging because nodes in the network may move and cause frequent and unpredictable topological changes. Thus, when two nodes travel apart, they may no longer have a direct link between them. Likewise, if a node moves behind a hill, its links to its neighbors may be severed because of fading. Other reasons for changes in topology include jamming and the entry of new nodes to the network or departure of the existing nodes from the network.

Location management enables an ad hoc network to track the locations of mobile terminals between call arrivals. Since mobile users are free to move within the coverage area, the network can only maintain the approximate location of each user. When a connection needs to be established for a particular user, the network has to determine the user's exact location within the defined granularity. Location management for an ad hoc network contains three components: location update, maintaining home regions, and locating a node. The operation of informing the home region about the current location of the mobile user is known as location update or location registration, and the messaging cost, measured in packets per second per node, of performing these activities is the cost of location update. When a node moves from region $A$ into region $B$, it needs to inform nodes in region $A$ of its departure and meanwhile, it needs to inform nodes in region $B$ of its arrival. It also needs to collect location information about all the nodes registered in region $B$. The operation of these activities is known as maintaining home regions, and the messaging cost of performing these activities is the cost of maintaining home regions. When a node receives a data packet for some destination, it needs to find the current location of the destination before sending packets to it. The operation of determining the location of the mobile user is called terminal locating or paging, and the messaging cost of performing 
these activities is the cost of locating a node.

Several approaches have been proposed to address ad hoc network routing and costs [1-6]. In [4] a scalable routing protocol is presented. This protocol relies on a location update mechanism that maintains approximate location information for all nodes in a distributed pattern. As nodes move, this approximate location information is constantly updated. To maintain the location information in a decentralized way, this paper maps a node ID to a geographic sub-region of the network. Any node present in this sub-region is then responsible for storing the current location of all the nodes mapped to this sub-region. In order to send packets to a node, the sender first queries the destination's sub-region for the approximate location of the destination, and then uses a simple geographic routing protocol to forward the packets to the destination's approximate location. It is therefore easy to see that the location update cost in this protocol is dependent on the speed of node movement.

SLALoM, a grid based location management scheme, scales well in large, mobile ad-hoc networks [5]. The scheme divides a square into some unit regions which is called order- 1 squares. It then combines $K^{2}$ of the order-1 squares to form order-2 squares. A node's home region will consist of an order-1 square. With some exceptions, every node has a home region in each order- 2 square. If an original square area is $A$, every node has $A / K^{2}$ home regions. The scheme partitions those home regions into near home region. By constructing the tree of home regions of a node based on some rules, the location update information of a node is easily disseminated by the span tree. When a node moves from one order- 1 to another, it not only sends a message to its nearby home regions, but also sends messages to the eight other home regions. Hence the location management cost is increased.

A novel multi-level hierarchical grid location management protocol with only one home region for a node that is called HGRID, for large scale ad hoc networks, is introduced in [6]. The paper shows that the average per node signaling cost in HGRID grows only logarithmically in the total number of nodes in a uniformly randomly distributed network - a substantial improvement over the signaling cost incurred by current location management schemes. If $S$ (source) and $D$ (destination) are co-located in the same grid, the location of $D$, as indicated by the location reply, is accurate and $S$ can forward the data directly to $D$ 's location. Otherwise, the location is approximate, and $S$ forwards the data packet to the location server specified in the location reply. When the data packet reaches the specified grid, the server $v$ that receives the packet checks its neighbor table to see if $D$ is co-located in the same grid. If so, the packet is successfully forwarded to the destination. Otherwise, the server searches for the $D$ in its location database. By construction, $v$ must have an entry for $D$ in its location database. If $v$ has accurate information about $D$, it further forwards the packet to $D$, otherwise, the packet is forwarded to the next location server which is in a level lower than $v$ in hierarchy, but has more accurate information about $D$ 's position. This process continues until the packet reaches $D$, or it reaches a lower grid, and the node that receives the packet drops it since it has no information about $D$. This can happen because $D$ would have left this grid, and $D$ 's location update to its new hierarchical leader failed to reach the leader before the data packet was forwarded by the leader to $D$ 's previously visited grid. Some times for location updating, the mobile node may inform two or more leaders, and those actions also increased the location management cost.

In order to decrease the location management cost, we propose a cooperative location management scheme, CooLMS, which is a nice integration of the grid model and pointer forwarding strategy. Pointer forwarding strategy was developed for location tracking in PCS networks. It helps reduce the cost of location update because when a node changes its location, it doesn't need to update its location information in its home region. It also helps reduce the cost of finding nodes because, with a forwarding pointer in place, a node can find out the location information of a destination node without the communication to the home region of the destination node. Pointer forwarding strategy has been recently studied quite intensively. Some variations have been proposed, such as a one-step painter forwarding strategy [13], location tracking pointer forwarding [14], two-level pointer forwarding strategy [15], K-step pointer forwarding strategy [16], and a combination of local anchor and forwarding pointer [17]. But to the best of our knowledge, nobody has ever introduced the pointers forwarding strategy into to the mobility management of mobile ad hoc networks. With the adoption of the pointer forwarding strategy, CooLMS reduces location update costs significantly.

In addition to the new effective location management scheme CooLMS, the paper also presents a more realistic location management cost model. Considering mobile users' movement is generally confined to a limited geographical area, the motion velocity of a mobile node is variable. Furthermore, the change of a mobile's velocity within a short time is limited due to physical restrictions. Therefore, a mobile user's future velocity is variable and likely to be correlated with its past and current velocity. The Gauss-Markov model [9] represents a wide range of user mobility patterns, including, as its two extreme cases, the random walk $[10,11]$ and the constant velocity fluid-flow models. Since it captures the essence of the correlation of a mobile's velocities in time, we use it to specify the characteristics of mobile node movement. 
Considering the measurements of mobile motion velocity are not necessarily consecutive, this paper presents an approach to the total cost discrete estimation of variable velocity mobile location management for ad hoc mobile networks.

The rest of the paper is organized as follows. In Section 2, we describe the cooperative location management scheme, which is based on grids and pointer forwarding strategy. In Section 3, we discuss the total cost of the cooperative location management scheme under constant velocity. Considering mobile users often travel at variable velocity, total location management cost estimation is presented based on a Gauss-Markov mobility model in Section 4. Simulation results which compare the performance of our CooLMS scheme with that of SLALoM and HGRID schemes are presented in Section 5. Section 6 concludes the paper.

\section{Cooperative Location Management Scheme}

In CooLMS, each node is assigned one and only one home region. All nodes in a home region act as the location server for any node in that region. This is different from schemes that select a single node in a region as the location server, in which the selected node can easily run out of battery.

In this section, we introduce CooLMS and explain its advantages over existing location management schemes.

\subsection{Selection of Service Regions}

We assume that mobile nodes are capable of knowing

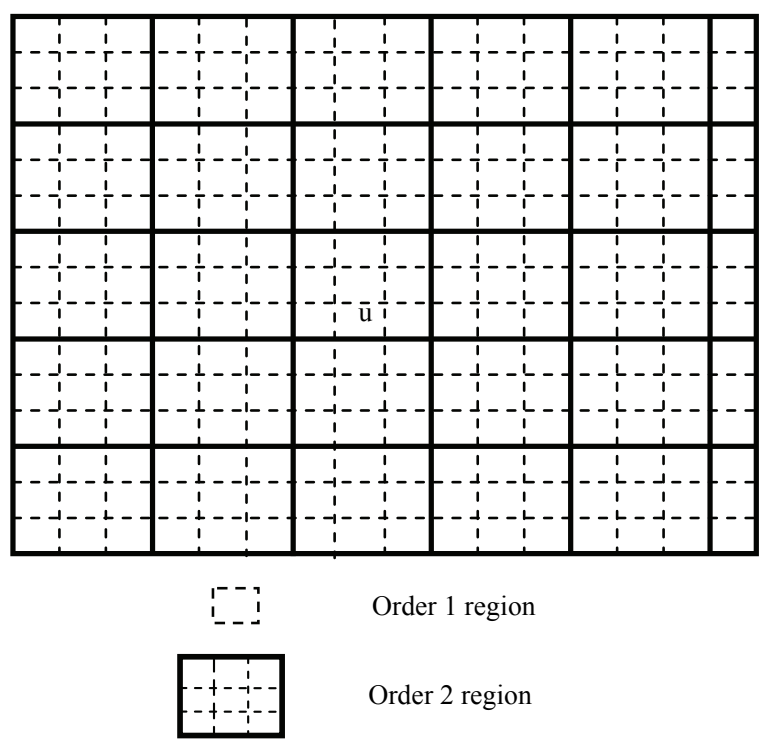

Figure 1. The location server region of a node. their current location, for example, using the Global Positioning System (GPS), and are equipped with radios with certain transmission range. We also assume the nodes move about in a square region of area $A$ so as to simplify the discussion. Our scheme, just like the one reported in [5], divides the square into unit regions which are called order-1 squares. It then merges every $K \times K$ order-1 square to form order-2 square. Figure 1 illustrates this idea, where solid line bordered squares are order- 2 squares and each order- 2 square contains $3 \times 3$ order-1 squares.

Using a predefined function $f$, each mobile node is assigned a single home region based on its ID, which is an order-1 region. The home region is also called service region. Notice that our grid model is different from the one proposed in SLALoM, in which every node has a home region in each order-2 square, hence every node has $O\left(A / K^{2}\right)$ home regions.

\subsection{Location Management Process}

The overhead cost of a location management scheme can be divided into three parts: location update cost, location maintenance cost and location finding cost. The location update cost covers all the signaling messages that nodes send to their home servers (in home region) whenever they move to a new location. The location maintenance cost covers all the signaling messages that nodes a) send to their previous order- 1 squares to inform them of their departure, b) send to their current order-1 squares to inform them of their arrival, and c) collect as they are now location servers for the nodes currently registered in their order-1 squares. The location finding cost covers all the signaling messages sent for locating a mobile node.
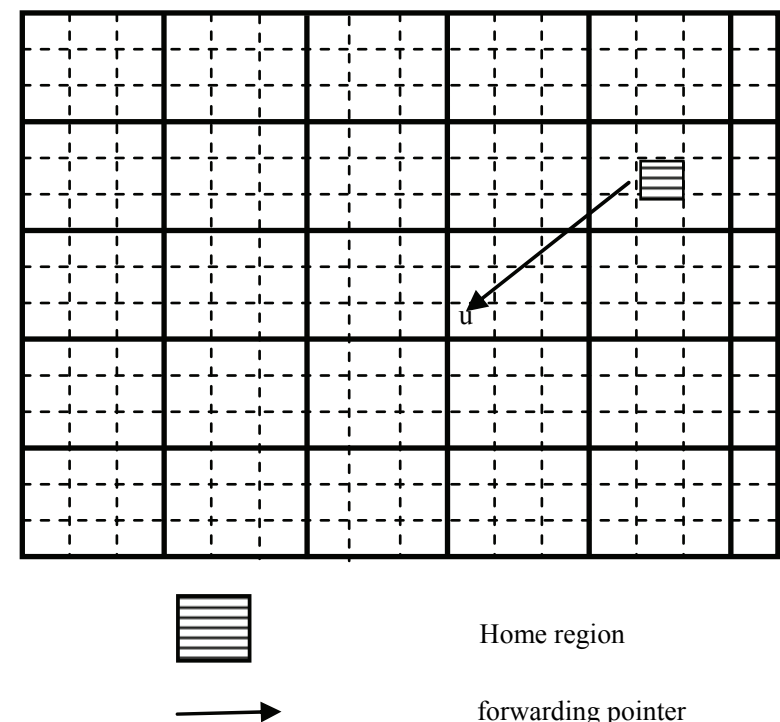

Home region

forwarding pointer

Figure 2. Home region and the forwarding pointer. 

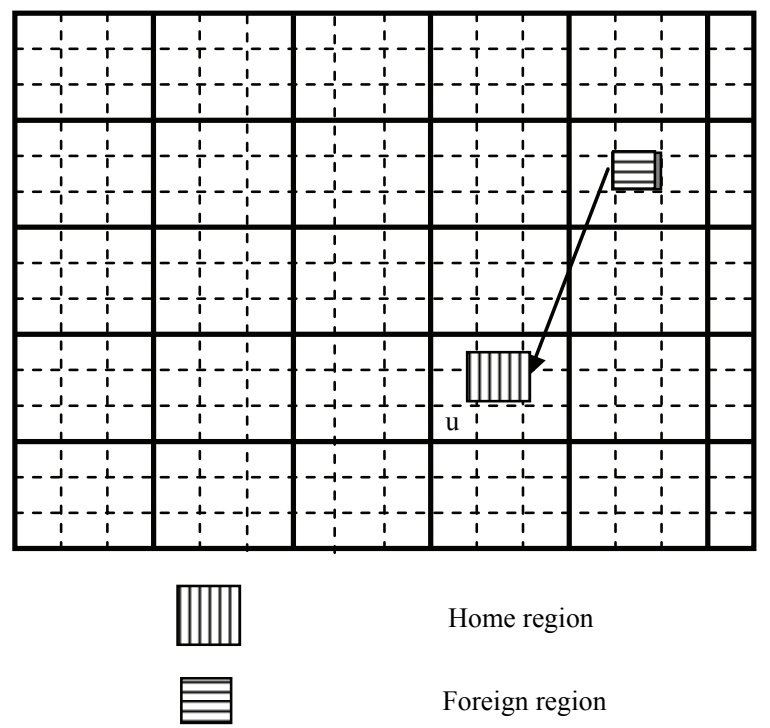

Figure 3. Home region and foreign region.

\subsubsection{Assigning Home Regions}

A predefined one-to-one mapping $f$ is used by the scheme to map the ID of a node to a region-1 square as its home region. This mapping is known to every node, so that each node can determine the home region of any other node in constant time.

\subsubsection{Maintaining Location with Forwarding Pointers}

When a node $u$ is in the network and located in the order-2 square of its home region, the home region knows the exact location information of node $u$. But when the node $u$ moves to one of the eight sibling order- 2 squares of the order-2 square of its home region, the home region may set a forwarding pointer to point to the order-1 square that node $u$ arrives, as illustrated in Figure 2.

If node $u$ is not in the eight order-2 squares nearby its home region, the home region authorizes the order- 2 square in which node $u$ resides to select an order-1 square as its foreign region, as shown in Figure 3. The home region of node $u$ knows roughly (not exactly) the location information of node $u$ in this situation. Just like the home region does, when the node $u$ further moves to one of the eight neighboring order- 2 squares of the order-2 square of its foreign region, the foreign region may set a forwarding pointer to point to the order-1 square that node $u$ arrives.

\subsubsection{Locating a Node}

When a source node $\mathrm{S}$ wants to send data packets to a destination node $\mathrm{D}, \mathrm{S}$ finds the home region of $\mathrm{D}$ using the D's ID and mapping $f$. It then sends a query packet to this home region to inquire of D's location. Four cases arise:

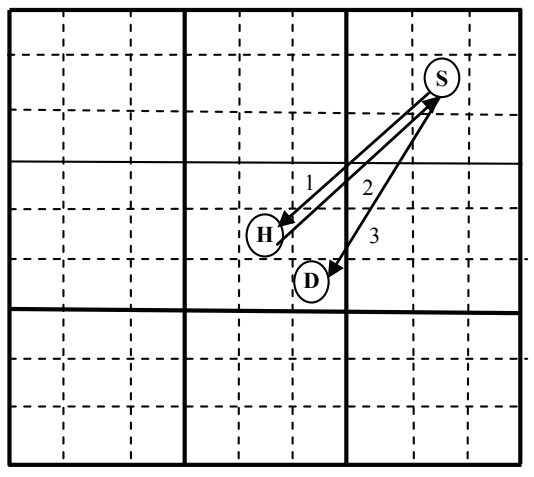

(a)

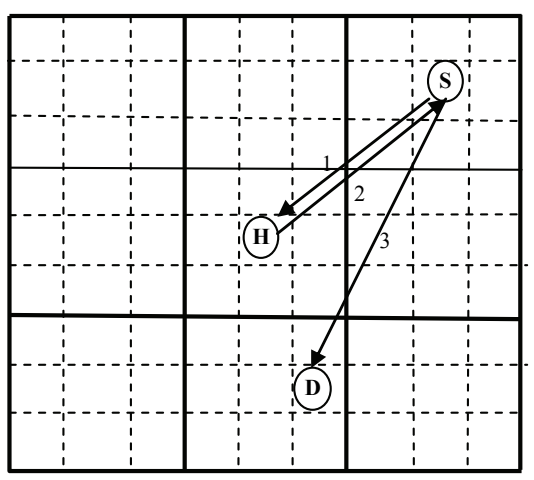

(b)

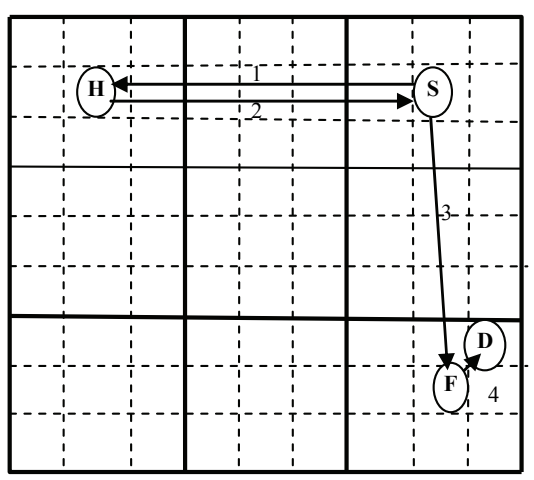

(c)

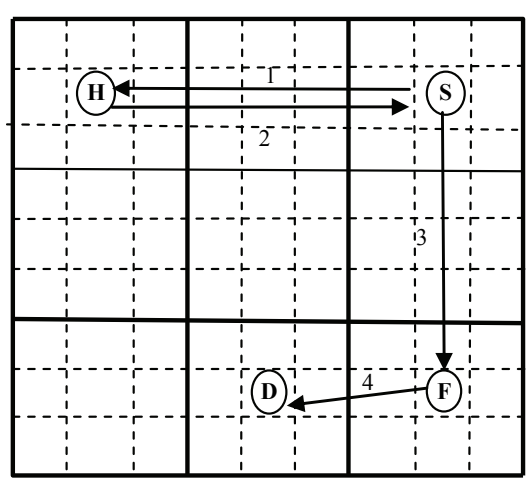

(d)

Figure 4. The location discovery process. (S: source node; D: destination node; $H$ : home region; $F$ : foreign region; $1,2,3$, and 4: the order of the location discovery process) 
1) If $\mathrm{D}$ is in the order-2 square of its home region, $\mathrm{S}$ requests the location information of destination node $\mathrm{D}$, the home region sends directly the location information of node D to source node S, and S can send directly the data packets to destination node D using this information, as shown in Figure 4(a).

2) If $D$ is in one of the eight neighboring order-2 squares to its home region order-2 square, $\mathrm{S}$ sends request to the home region of $\mathrm{D}$. $\mathrm{S}$ can send directly the data packets to destination node D using this information, as shown in Figure 4(b)

3 ) If $D$ is in the order- 2 square of its foreign region, the home region sends the location information of the foreign region to source node $\mathrm{S}$, and $\mathrm{S}$ requests D's foreign region for D's location information. After this process, the data packets from $\mathrm{S}$ can be retransmitted by the home region and foreign region to $D$, as shown in Figure 4(c).

4) If $D$ is in one of the eight sibling order- 2 squares to its foreign region order- 2 square, $\mathrm{S}$ sends data packets to the foreign region, and the foreign region retransmit the packets by forwarding pointer to destination node $\mathrm{D}$, as shown in Figure 4(d).

\subsection{Forwarding Pointers}

When mobile node $u$ leaves the order-2 square of its home region, the home region sets a forwarding pointer pointing to the order- 1 square where $u$ arrives. The forwarding pointer is called first class forwarding pointer, and the order- 1 square that node $u$ arrive is called a foreign region. When $u$ further moves to another sibling order-1 square as shown in Figure 5, the previous order-1 square can generate a forwarding pointer pointing to the new order-1 square where $\mathrm{u}$ is currently located; it
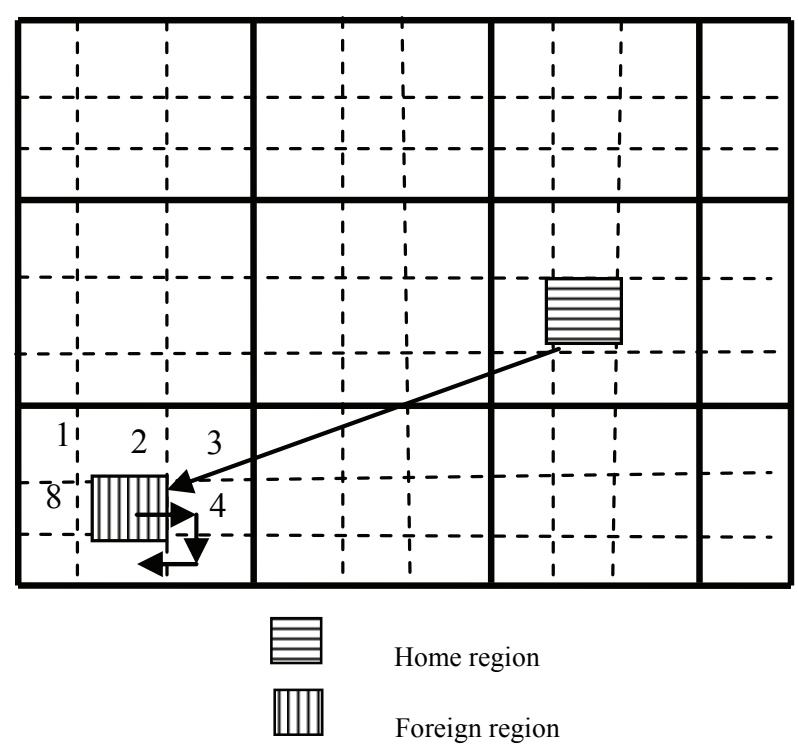

Figure 5. The process of setting up forwarding pointers. doesn't need to inform its home region. This reduces the updating and paging packets cost. We call the forwarding pointer generated by order-1 squares the second class forwarding pointer.

We illustrate the process of setting up forwarding pointers in Figure 5. The square filled with horizontal strips is the home region of node $u$, while the square filled with vertical strips is its foreign region. The arrow from the home region to the foreign region is the first class pointer, while the arrows around the foreign region are the second class forwarding pointers. If $u$ moves from the foreign region to the square numbered 4 , a second class forwarding pointer is set to indicate the current location of $u$. Similarly, if it moves to the square numbered 5 from the one numbered 4, another second class forwarding pointer is set pointing to the square numbered 5 , and so on.

We see from the process that increasing the number of forwarding pointers will reduce the location updating cost, but may also increase the paging cost. It is a tradeoff phenomenon in location management. If a node moves across many region-2 square, we can further setup forwarding pointers from one foreign region to another around its home region, just like the process of setting up forwarding pointers from one order-1 region to another neighboring foreign region as shown in Figure 5. We will discuss the optimal number of forwarding pointers in Section 5 .

\section{The Cost of Cooperative Location Management}

In this section, we discuss how to evaluate the total location management cost, which is measured in packets per second per node to transfer. We assume that nodes distribute uniformly, move randomly and independently of each other. Each node selects a direction to move, chosen uniformly between $[0,2 \pi]$. Each node selects its speed, chosen uniformly between $[v-c, v+c]$ for some time $t$, where the $t$ is exponentially distributed with a mean of $\tau$. After a mobile has traveled for time $t$, it selects another direction, speed and time to travel. The mobility model and the geographic routing algorithm, MFR (most forward with fixed radius routing, which forwards packets to the neighbor closest to the destination node) [12] is the same as in [5] and [6], which allows us to compare our scheme's performance with [5] and [6].

We also assume that the ad hoc network under study is in a rectangular region; all nodes in the network are equipped with Global Positioning System (GPS) that provides them with their current location; they are aware of the identities of their neighbors; and each node has a unique ID (such as an IP address). We use the following notations in this section: 
$A$ area of the total networks

$N$ number of nodes

$M$ the threshold of forwarding pointer number

$K$ network area $A$ is divided into $K^{2}$ order-1 squares

$a$ area of a order-1 region

$b$ broadcast cost in a region

$v$ speed (meters per second)

$u$ cost of sending location update message to home region

$\delta$ cost of collecting location information

$\gamma$ average density of nodes in ad hoc networks

$r$ radius of a node

$z \quad$ average distance for one hop of a node

$\lambda$ Poisson distribution parameter of the data packets arrive at

As mentioned in Section 2, the network area is partitioned into unit regions or squares. Based on the distribution and mobility assumption made in the beginning of the section, the size of the unit region is chosen so that its average node density $\gamma$ is approximately a constant. Thus, the total area of the networks $A=N / \gamma$, Woo and Singh [4] noted the following:

1) The cost of broadcasting in an order-1 square by a node, $b$, is proportional to the number of transmissions needed to cover the said square. The latter is in turn proportional to the area of the order-1 square divided by the area covered by a single transmission, and also is the location updating cost for just only time. Thus, $b=$ $O\left(a / r^{2}\right)$.

2) The distance a node $u$ has to cover to cross an order- 1 square is proportional to the side of an order-1 square. Thus, the number of order-1 squares that a node crosses per second, is proportional to $n_{1}=O(v / \sqrt{a})$.

3) Similarly, the number of order-2 squares that a node $u$ crosses per second, can also be estimated by $n_{2}=O\left(n_{1} / K\right)=O(v / K \sqrt{a})$ order-1 squares per second.

4) The cost of updating all severing region include foreign regions and order-1 squares estimated by $n_{3}=O\left(n_{1} / M\right)=O(v / M \sqrt{a})$.

\subsection{Cost of Location Update}

When a node $u$ moves out of the current region and enters a new region, three cases of location update may occur:

1) When the new region locates in the order-2 square of its home region, the home region knows the exact location information of node $u$. In this case, we only broadcast the $n_{1}$ order- 1 nodes. The location update cost is $O\left(n_{1}(b)\right)$.

2) When the node $u$ moves to one of the other neighboring eight order-2 squares of its home region order-2 square, the home region may set a forwarding pointer to point the order-1 square that node $u$ arrives. The location update cost is $O\left(n_{2}(b+K / z)\right)$.

3) When the node $u$ is not in the nine order- 2 squares nearby its home region discussed above, the home region authorizes the order- 2 square where the node $u$ locates to select an order-1 square as a foreign region of the node $u$. The home region of node $u$ knows roughly the location information of node $u$ in this situation. Just like the home region does, when the node $u$ moves to one of the other eight order-2 squares (not include the order-2 square the foreign region locates) that neighbor to the order-2 square of its foreign region, the foreign region may set a forwarding pointer to point to the order-1 square that node $u$ arrives. The location update cost is $O\left(n_{3}\right.$ $\left.\left(A b / K^{2}+A / K\right)\right)$.

So, the total location updating $\operatorname{cost} c_{u}$ is given by

$$
\begin{aligned}
& c_{u}=O\left(n_{1}(b)+n_{2}(b+K / z)+n_{3}\left(A b / K^{2}+A / K\right)\right) \\
& =O\left(\frac{v}{\sqrt{a}} \frac{a}{r^{2}}+\frac{v}{K \sqrt{a}}\left(\frac{a}{r^{2}}+\frac{K}{z}\right)+\frac{v}{M \sqrt{a}}\left(\frac{N}{\gamma} \frac{a}{r^{2}} \frac{1}{K^{2}}+\frac{N}{\gamma} \frac{1}{K}\right)\right) \\
& =O\left(v+\frac{v}{K}+\frac{v N}{M K}+\frac{v N}{M K^{2}}\right)
\end{aligned}
$$

\subsection{Cost of Maintaining Location Information}

There is no such a cost in cell phone network because of the existence of base stations. However, in mobile ad hoc networks, when a node moves from region $A$ into region $B$, it needs to inform nodes in region $A$ that it has left and meanwhile, it needs to inform nodes in region $B$ of its arrival. The nodes need to take three steps to maintain location information, namely

1) Inform the original order-1 region of its departure;

2) Inform the new order-1 region of its arrival;

3) As a location server in the new order-1 region, cache the registration information of nodes in the region.

So the cost for maintaining nodes location information $c_{m}$ is

$$
\begin{aligned}
& c_{m}=O\left(n_{1}(b+b+\delta)\right)=O\left(n_{1}(2 b+\delta)\right) \\
& =O\left(\frac{v}{\sqrt{a}}\left(\frac{2 a}{r^{2}}+\delta\right)\right)=O(v)
\end{aligned}
$$

\subsection{Cost of Finding Nodes}

The cost of finding the location might be zero or a constant if either the node itself or one of its neighbors has the location information available in a cache, which happens if there are several packets destined for the same destination. In our analysis, however, we make the pessimistic assumption that the location information is not cached; therefore, the source node needs to contact the destination node's home region to find the destination's location. 
The location finding process includes two situations (combined from the four cases described in Subsection 2.2.3)

1) When the source node A wants to send data packets to its destination $\mathrm{B}$, node $\mathrm{A}$ queries the home region of node $\mathrm{B}$. If node $\mathrm{B}$ is in its home region, then node $\mathrm{A}$ directly sets up a linkage with node B. From Figure 4 (a) we can deduce the cost is asymptotically to $3 d(\mathrm{~S}, \mathrm{H}) / z$, where $d(\mathrm{~S}, \mathrm{H})$ is the distance between the source node $\mathrm{S}$ and the home region, and it is proportional to $K$. So we know that the location finding cost is proportional to $K$. Similarly, from Figure 4 (b) we can also conclude that the location finding cost is proportional to $K$;

2 ) If node $B$ is not in its home region, then node A sets up a linkage through B's home region, the first and second class forwarding pointers, and then sends packets using this linkage. From Figure 4 (c) and (d), the location finding cost is asymptotically to $[2 d(\mathrm{~S}, \mathrm{H})+d(\mathrm{~S}, \mathrm{~F})] / z$, where the $d(\mathrm{~S}, \mathrm{~F})]$ is the distance between the source node $\mathrm{S}$ and the foreign region, and it is proportional to $K$. For forwarding pointer cost, we can easily get that it is proportional to $M$ and $\lambda$.

So the average total cost of locating a node is

$$
c_{d}=O((K / z)+O(\lambda M / z)
$$

\subsection{The Total Cost of Location Management}

The total cost of location management

$$
\begin{aligned}
& c_{\text {total }}=N\left(c_{u}+c_{m}+c_{d}\right) \\
& =O\left(2 v N+\lambda N M+K N+v N / K+v N^{2} /(K M)+v N^{2} /\left(K^{2} M\right)\right)
\end{aligned}
$$

\section{Gauss-Markov Model Based Total Location Management Cost Estimation}

\subsection{Gauss - Markov Mobility Model}

Mobile nodes often have to change speed during the course of motion. We assume that mobile nodes move at inconstant velocity and the velocity change follows a Gauss-Markov process. According to [9], the 1-D discrete version of the Gauss-Markov mobility model can be described as:

$$
v_{n}=\alpha v_{n-1}+(1-\alpha) \mu+\sigma \sqrt{1-\alpha^{2}} w_{n-1}
$$

where $v_{n}$ is a node's mobile velocity during the $n$-th period, $\alpha$ is the memory level, which reflects the relationship between $v_{n-1}$ and $v_{n}, \mu$ the mean of $v_{n}$, $\sigma^{2}$ the variance of $v_{n}$, and $w_{n}$ an uncorrelated Gaussian process with zero mean, unit variance. $w_{n}$ is independent of $v_{n}$.
Let $u_{n}=v_{n}-\mu, \beta=\sigma \sqrt{1-\alpha^{2}}$, the Equation (2) can be rewritten in the following simple and clear form

$$
u_{n}=\alpha u_{n-1}+\beta w_{n}
$$

\subsection{Total Location Management Cost}

After we have estimated each of the individual costs involved in location updating information, maintaining location information and finding the location information, we can estimate the total cost of routing packets. Assume that packets arrive at each node at a rate of $\lambda$ packets per second according to a Poisson process. Then the average cost of routing $\mathrm{N}$ nodes can be calculated as (1). Recall the assumption that mobile motion process is GaussMarkov. Let

$$
v_{n}=u_{n}+\mu
$$

We know from Section III that the location updating cost $c_{u}=O(v)=K_{1} v$, the maintaining location cost $c_{m}=$ $O(v)=K_{2} v$, and the finding node cost is constant $K_{3}$. So the total cost $c_{\text {total }}=\left(K_{1}+K_{2}\right) v+K_{3}$. Substituting $v$ with (4), we obtain

$$
\begin{aligned}
c_{\text {total }}= & \left(K_{1}+K_{2}\right)\left(u_{n}+\mu\right)+K_{3} \\
& =\left(K_{1}+K_{2}\right) u_{n}+\left(K_{1}+K_{2}\right) \mu+K_{3}
\end{aligned}
$$

Let

$$
\begin{aligned}
& \eta=K_{1}+K_{2} \\
& \kappa=\left(K_{1}+K_{2}\right) \mu+K_{3}
\end{aligned}
$$

Then it follows from (5) and (6) that

$$
c_{n}=\eta u_{n}+\kappa
$$

From (3), we have

$$
u_{n}=\alpha^{n} u_{0}+\left(\sum_{k=0}^{n-1} \alpha^{k} w_{n-k}\right) \beta
$$

where the $u_{0}$ is the initial value of $u_{n}$. It results from (7) that

$$
c_{n}=\eta\left[\alpha^{n} u_{0}+\left(\sum_{k=0}^{n-1} \alpha^{k} w_{n-k}\right) \beta\right]+\kappa
$$

Consider $w_{n}$ is an uncorrelated Gaussian process with zero mean, unit variance, and $w_{n}$ is independent of $v_{n}$, then mean and variance of $c_{n}$ are given as

$$
\begin{aligned}
& E c_{n}=u_{0} \eta \alpha^{n}+\kappa \\
& D c_{n}=\sum_{k=0}^{n-1} \alpha^{2 k} \beta^{2} \sigma^{2}=\frac{\beta^{2} \alpha^{2}\left(1-\alpha^{2 n}\right)}{1-\alpha^{2}}
\end{aligned}
$$


When the memory level $0<\alpha<1$, we have

$$
\begin{aligned}
& \lim _{n \rightarrow+\infty} E c_{n}=\kappa \\
& \lim _{n \rightarrow+\infty} D c_{n}=\frac{\beta^{2} \alpha^{2}}{1-\alpha^{2}}
\end{aligned}
$$

We give approximate estimation of the total cost of location management in this section, and we will give some simulation results in the next section.

\section{Simulation Results}

In this section, we present the simulation results for CooLMS, SLALoM [5] and HGRID [6] in network time delay and total location management cost, using OPNET technology [18].

To compare the performance of these three schemes, a location management layer was built in to the TCP/IP protocol stack that is operated in conjunction with IP as the network layer protocol. Main data structures [6] in the location management layer consist of a location table

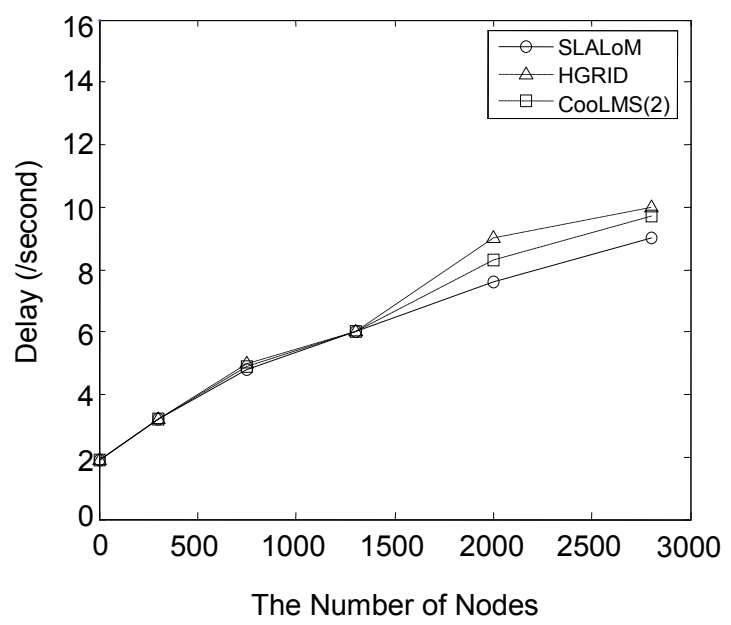

(a) $\mathrm{M}=2$ for time delay

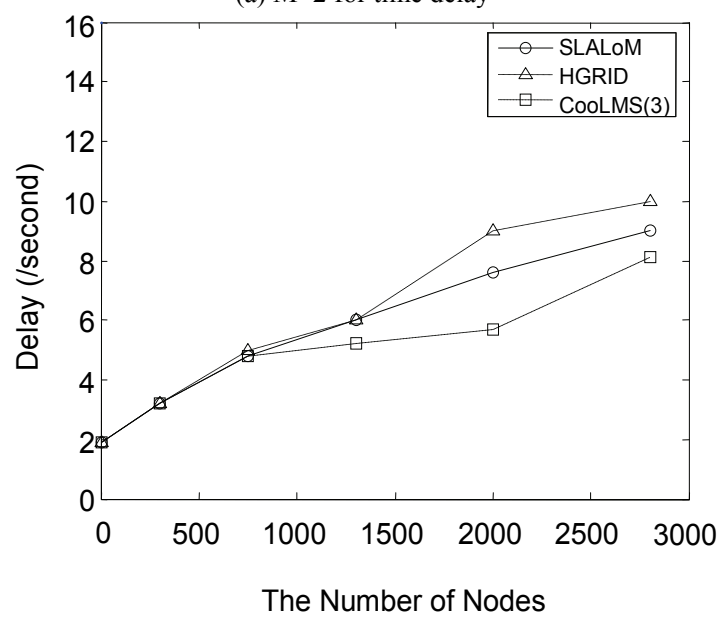

(b) $\mathrm{M}=3$ for time delay

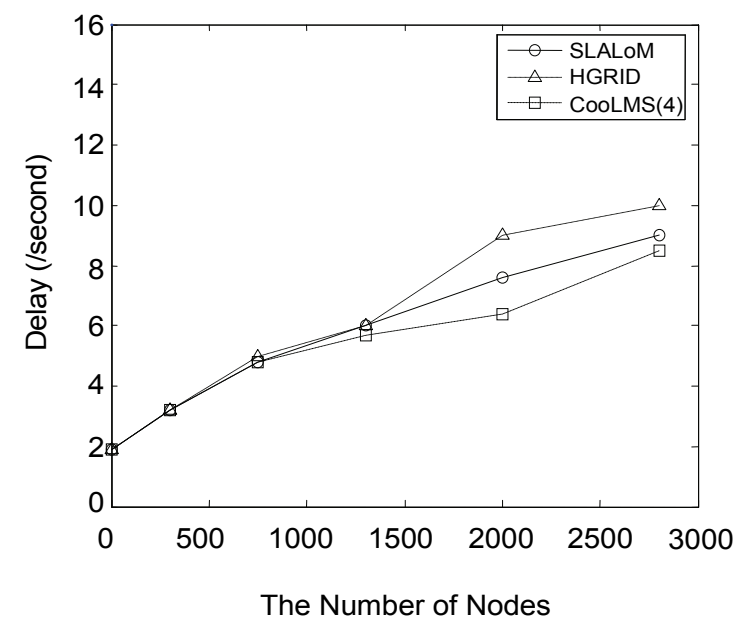

(c) $\mathrm{M}=4$ for time delay

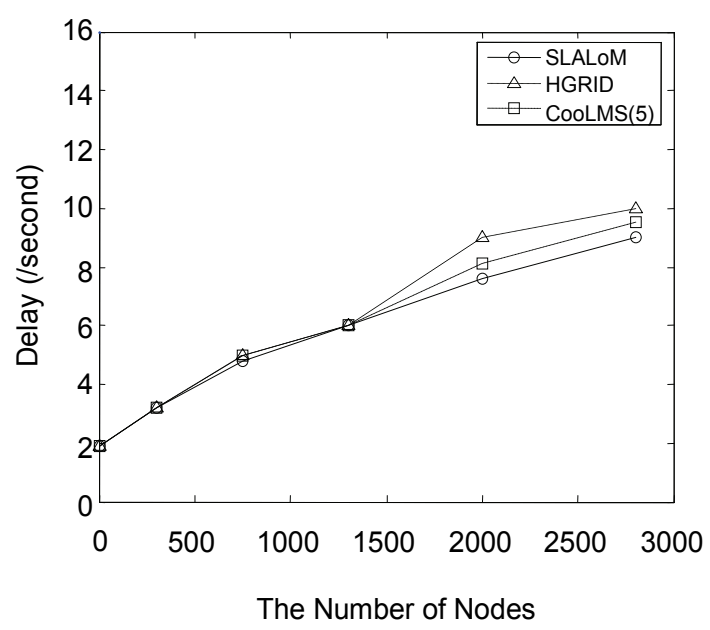

(d) $\mathrm{M}=5$ for time delay

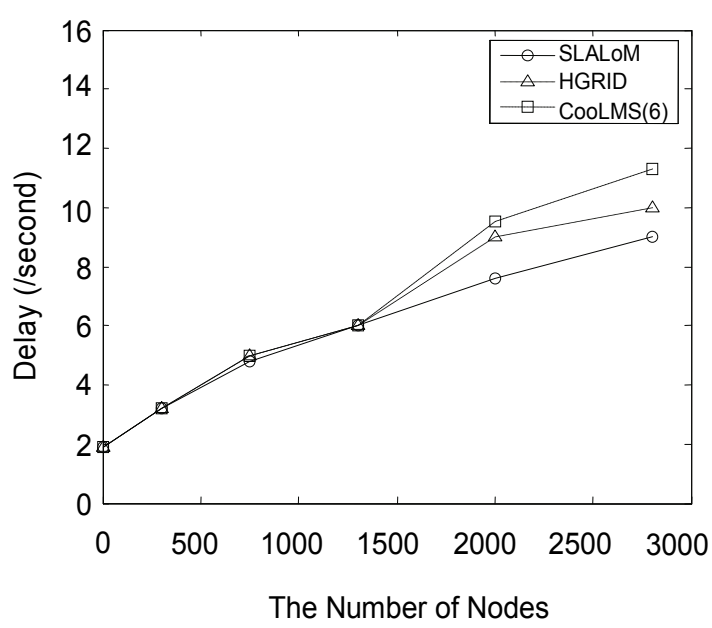

(e) $\mathrm{M}=6$ for time delay

Figure 6. Network time delay.

and a neighbor table. When a location server node receives a location update packet from a node, the current location of that node is updated in the location table. 
MFR [12] without backward progression, in which packets are dropped if no forward progress can be made, was implemented as the geographic routing algorithm.

We assume that nodes distribute uniformly, move randomly and independently of each other. Each node selects a direction to move, chosen uniformly between $[0,2 \pi]$.

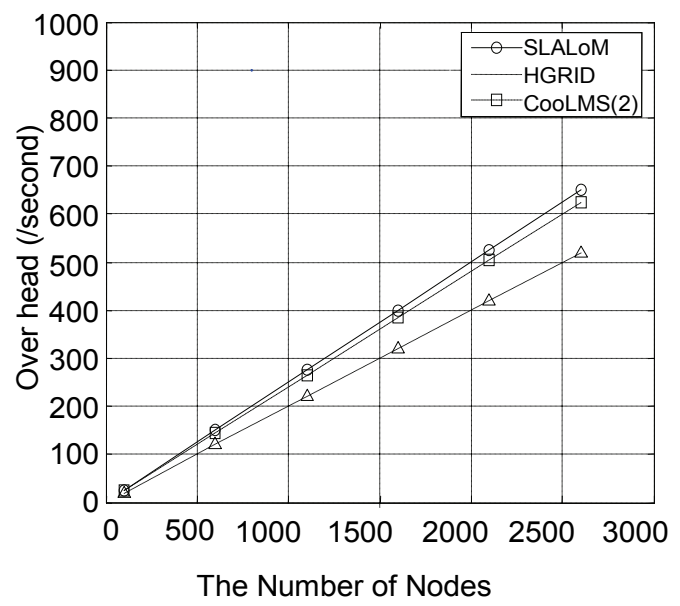

(a) $\mathrm{M}=2$ for overhead

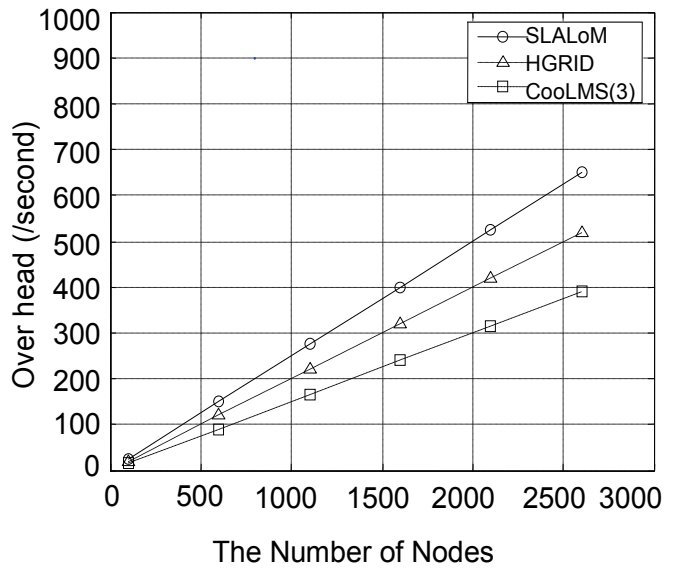

(b) $\mathrm{M}=3$ for overhead

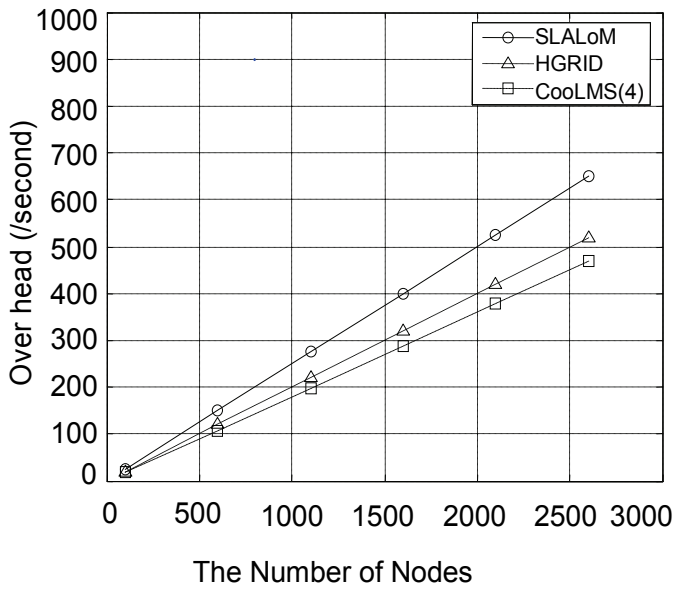

(c) $\mathrm{M}=4$ for overhead

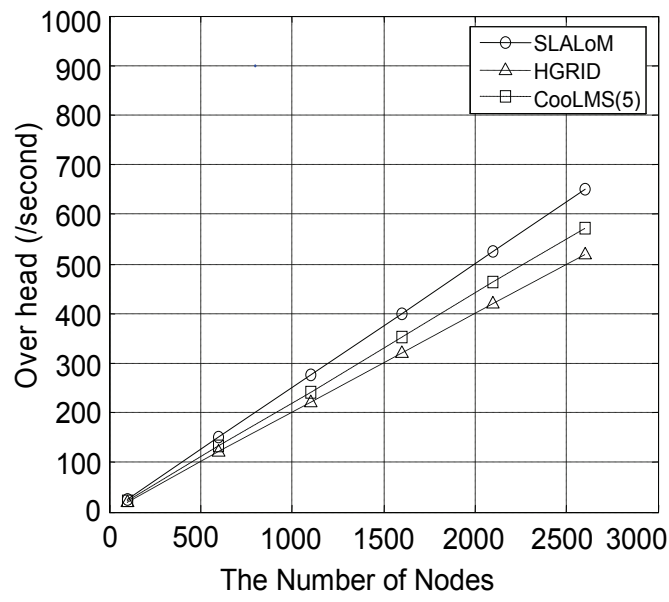

(d) $\mathrm{M}=5$ for overhead

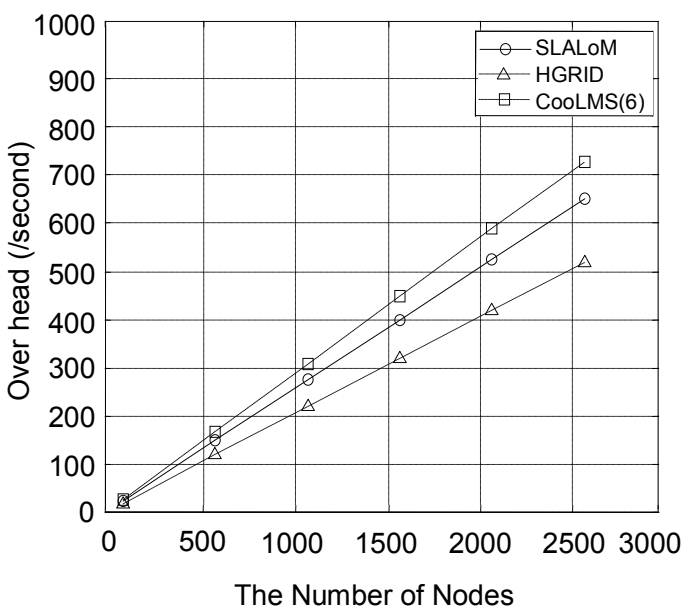

(e) $\mathrm{M}=6$ for overhead

Figure 7. Location management overhead.

Each node selects its speed, chosen uniformly between [0, $10 \mathrm{~m} / \mathrm{s}$ ] for some time $t$, where $t$ is exponentially distributed with mean $\tau$. After a mobile has traveled for certain time $t$, it selects another direction, speed and time to travel. The topology consists of from 50 to 3000 nodes. When the number of nodes increase, the areanetwork time delay and total location management cost. As shown in Figure 6, there is a tradeoff between paging, updating cost and network time delay. We can balance the tradeoff by selecting the threshold of forwarding pointer number. When threshold of forwarding pointer number $M=3$ and 4 , the network time delay with CooLMS is lower than the networks with SLALoM and HGRID; when threshold of forwarding pointer number $M=2$ and 5, the network time delay with CooLMS is between the networks with SLALoM and HGRID; When threshold of forwarding pointer number $M=6$, the network time delay with CooLManet is higher than the networks with SLALoM and HGRID.

Figure 7 shows that when the threshold of forwarding pointer number $M=3$ and 4 , the total location manage- 
ment cost with CooLManet is lower than the networks with SLALoM and HGRID; When the threshold of forwarding pointer number $M=2$ and 5 , the total location management cost with CooLMS is between the networks with SLALoM and HGRID; When the threshold of forwarding pointer number $M=6$, the total location management cost with CooLManet is higher than the networks with SLALoM and HGRID.

We conclude from the simulation that using suitable number of forwarding pointers including the first and second pointers, the network time delay and location management cost can be reduced significantly.

\section{Conclusions}

We proposed a cooperative location management scheme, CooLMS, for mobile ad hoc networks. CooLMS combines the strength of grid based location management and cooperative location management to achieve low signaling cost as well as high scalability. We also discussed the total cost estimation of mobile location management for ad hoc mobile networks with missing measurements. Simulation results indicate that the network time delay and location management cost can be reduced significantly by using suitable number of forwarding pointers. Practically, the CoolMS scheme is good for networks used in metropolis areas where mobile users typically travel across several location areas from home to work on a daily basis.

Our future work includes location management for special routing protocols and location management for QoS of mobile decision support in ad hoc mobile network.

\section{Acknowledgment}

This work is partially supported by NSFC granted number 60874113 and 70271001; China Postdoctoral Fund granted number 2002032191; Shanghai Fund of Science and Technology granted number 00JG05047; Shanghai Key Scientific Research Project under grant number 05dz05036; and 2008 Fund of Engineering Research Centre of Digitized Textile \& Fashion Technology, Ministry of Education.

\section{References}

[1] J. Broch, D. A. Maltz, D. B. Johnson, Y.-C. Hu, and J. Jetcheva, "A performance comparison of multi-hop wireless ad hoc network routing protocols," Proceedings of 4th Annual Conference on Mobile Computing and Networking, Dallas, TX, October 25-30, 1998.

[2] T.-W. Chen and M. Gerla, "Global state routing: A new routing scheme for ad-hoc wireless networks," Proceedings of IEEE International Communications Conference, 1998.

[3] Y.-B. Ko and N. H. Vaidya, "Location-aided routing
(LAR) in mobile ad hoc networks," Proceedings of 4th Annual Conference on Mobile Computing and Networking, Dallas, TX, October 25-30, 1998.

[4] S. M. Woo and S. Singh, "Scalable routing protocol for ad hoc networks," Wireless Networks, Vol. 7, pp. 513529, 2001.

[5] C. Cheng, H. Lemberg, S. Philip, E. van den Berg, and Tao Zhang, "SLALoM: A scalable location management scheme for large mobile ad-hoc networks," Proceedings of IEEE Wireless Communications and Networking Conference, Vol. 2, pp. 574-578, March 2002.

[6] S. J. Philip, J. Ghosh, and C. M. Qiao, "Performance evaluation of a multilevel hierarchical location management protocol for ad hoc networks," Computer Communications, Vol. 28, pp. 1110-1122, 2005.

[7] S. M. S. Masajedian and H. Khoshbin, "Cooperative location management method in next generation cellular networks," Proceedings of the Ninth International Symposium on Computers and Communications, pp. 525-530 2004.

[8] J. Zhou, B. Tang, and D. Li, "Partition digraph for location area management in mobile computing environment," International Journal of Nonlinear Sciences and Numerical Simulation, Vol. 5, No. 4, pp. 393-396, 2004.

[9] B. Liang, and Z. J. Haas, "Predictive distance-based mobility management for multidimensional PCS networks," IEEE/ACM Transactions on Networking, Vol. 11, No. 5, pp. 718-732, 2003

[10] J. S. M. Ho and I. F. Akyildiz, "Mobile user location update and paging under delay constraints," ACM-Baltzer J. Wireless Networks, Vol. 1, pp. 413-425, December 1995.

[11] Y.-B. Lin, "Reducing location update cost in a PCS network," IEEE/ACM Trans. Networking, Vol. 5, pp. 25-33, February 1997.

[12] T.-C. Hou and V. O. K. Li, "Transmission range control in multihop packet radio networks," IEEE Transactions on Communications, Vol. COM-34, No. 1, pp. 38-44 1986.

[13] K. Sue and C. Tseng, "One-step pointer forwarding strategy for location tracking in distributed HLR environment," IEEE Journal on Selected Areas in Communications, Vol. 15, No. 8, pp. 1455-1466, 1997

[14] Y. Lin and W. Tsai, "Location tracking with distributed HLR's and pointer forwarding," IEEE Transaction on Vehicular Technology, Vol. 47, No. 1, pp. 58-64, 1998

[15] W. Ma and Y. Fang, "Two-level pointer forwarding strategy for location management in PCS networks," IEEE Transaction on Mobile Computing, Vol. 1, No. 1, pp. 32-45, 2002

[16] C.-M. Weng and C.-H. Chu, "K-step pointer forwarding strategy for location tracking in distributed HLR environment," IEE Proceedings of Communications, Vol. 150, No. 3, pp. 207-213, June 2003.

[17] W. Ma and Y. Fang, "An efficient mobility management scheme based on location anchoring and pointer forwarding," IEEE $58^{\text {th }}$ Vehicular Technology Conference, VTC'03-Fal1, Vol. 4, pp. 2764-2768,6-9 October 2003.

[18] http://www.opnet.com/solutions/network_rd/modeler.html. 